\title{
Colonization Dynamics and Spatial Progression of Verticillium dahliae in Individual Stems of Two Potato Cultivars with Differing Responses to Potato Early Dying
}

\author{
J. Bae, Department of Horticulture, University of Wisconsin-Madison, 53706; Z. K. Atallah, Department of Plant \\ Pathology, University of Wisconsin-Madison, 53706; S. H. Jansky, USDA-ARS Vegetable Crops Research Unit; \\ and D. I. Rouse and W. R. Stevenson, Department of Plant Pathology, University of Wisconsin-Madison, 53706
}

\begin{abstract}
Bae, J., Atallah, Z. K., Jansky, S. H., Rouse, D. I., and Stevenson, W. R. 2007. Colonization dynamics and spatial progression of Verticillium dahliae in individual stems of two potato cultivars with differing responses to potato early dying. Plant Dis. 91:1137-1141.

Potato early dying (PED), caused by Verticillium dahliae, is a chronic yield-limiting disease of potato (Solanum tuberosum). In this study, we describe the colonization dynamics of V. dahliae in two potato cultivars with varying responses to PED. We utilized a quantitative real-time polymerase chain reaction (Q-PCR) assay to assess the colonization and spatial progression of $V$. dahliae in cvs. Ranger Russet (moderately resistant) and Russet Norkotah (highly susceptible). Ninety plants per cultivar were inoculated with a conidial suspension in the greenhouse. Every 2 weeks until week 10, we collected basal samples from 15 plants, and repeatedly sampled the growing apices of another 15 plants. The mean infection coefficient (IC) values in the basal and apical samples were significantly lower in cv. Ranger Russet at all five sampling dates. The pathogen was detected in basal samples of both cultivars by week 2 , and in apical samples of cv. Russet Norkotah at week 4 and of cv. Ranger Russet at week 6. Colonization of cv. Russet Norkotah consistently increased in apical and basal samples during the 10 weeks, while it plateaued after week 6 in cv. Ranger Russet. Differences in response to PED appear associated with the speed of colonization and the establishment of a higher population density by $V$. dahliae in the plant.
\end{abstract}

Potato early dying (PED), or Verticillium wilt, is a chronic disease of potato (Solanum tuberosum) and a leading chronic yield-limiting factor in irrigated production regions worldwide (24-26). PED is primarily caused by the prevalent soilborne fungus Verticillium dahliae and is often involved in a synergistic combination with the nematode Pratylenchus penetrans, but could also be caused by V. alboatrum in cooler growing regions (14,16,24-26). The disease leads to a reduction in photosynthetic activity (4) and gas exchange (5) of leaves, and premature senescence of potato plants, which shortens the period of tuber bulking, subsequently reducing yields (29). Yield losses associated with PED may reach 50\%, but are more commonly in the range of 10 to $15 \%$ (24-26).

Verticillium wilt is a monocyclic disease, and the pathogen survives between growing seasons as microsclerotia, which are dispersed on seed tubers, farm equip-

Corresponding author: Zahi K. Atallah
E-mail: atila@plantpath.wisc.edu

J. Bae and Z. K. Atallah contributed equally to this work.

Accepted for publication 2 April 2007.

doi:10.1094/PDIS-91-9-1137

(C) 2007 The American Phytopathological Society ment, by wind currents, and in soil and water. Currently, PED is mainly controlled through fumigation in fields previously grown to potato or by planting in fields never previously planted with potato ("virgin land"). A few potato cultivars, such as Ranger Russet and Bannock Russet, exhibit useful levels of resistance to $V$. dahliae in the field, but they currently represent a growing percentage of the total U.S. area planted to potato, the majority of which is planted to susceptible cultivars (for example Russet Burbank, Russet Norkotah). Several wild and interspecific hybrids of Solanum species show significant levels of resistance to PED and are currently being investigated as sources of resistance (1113,18).

Cultivars Russet Norkotah (highly susceptible) and Ranger Russet (moderately resistant) are commercially grown in the United States and Canada and represent among commercial cultivars opposite ends of the spectrum of response to $V$. dahliae and PED. Cultivar Russet Norkotah, released in 1987, was selected from a cross between ND9526-4 Russ and ND9687-5 Russ, which have Nooksack (resistant) and Norgold Russet (susceptible) in their pedigrees (15). Russet Norkotah is a mediumlate maturing cultivar (90 to 110 days) producing higher yields than Russet Burbank, but is mainly used for the fresh market. Cultivar Ranger Russet, a cross between Butte (moderately susceptible) and
A6595-3 (resistant), was released in Idaho in 1991 (23) as an alternative to cv. Russet Burbank. This cultivar carries resistance to potato early dying, Potato virus $X$ (PVX), Potato virus $Y$ (PVY), Potato leafroll virus (PLRV) net necrosis, and Fusarium dry rot. Similar to cv. Russet Burbank, it is late maturing (more than 130 days) and is mainly used for French fry processing.

Little information is available regarding the colonization dynamics by $V$. dahliae in potato cultivars during a single growing season. Nicot and Rouse (22) found a linear logit relationship "with a large random error" between soil inoculum density and stem colonization over four sampling dates during a single growing season. Nevertheless, numerous studies have only focused on the relationship between colonization/symptoms at the end of a growing season and yield $(1,7,10,20,29)$, while Davis et al. (7) studied colonization over an entire growing season. The quantification of $V$. dahliae in plant tissues is commonly conducted using semi-selective culture media such as NPX (6). The number of colonies observed on the NPX medium 2 weeks after transfer is translated into the number of CFU, which is used as a quantitative measure of stem colonization. Atallah et al. (2) described a quantitative real-time polymerase chain reaction (QPCR) assay for the detection and quantification of $V$. dahliae and were able to detect as few as five nuclei of the fungus in plant material, mainly stems. The assay improved detection sensitivity and reduced quantification variability, which affect plating methods $(19,21,27)$.

This study aims to describe the dynamics of colonization by $V$. dahliae in two potato cultivars with varying responses to PED, in an attempt to provide breeders with a new approach for the selection of resistance to PED.

\section{MATERIALS AND METHODS}

Greenhouse inoculations. Nuclear seed, $V$. dahliae free, of cvs. Ranger Russet (moderately resistant) and Russet Norkotah (highly susceptible) were planted in the greenhouse on 10 February 2006 (trial 1) and 7 April 2006 (trial 2) in a 1:1 vol/vol vermiculite and commercial soilless potting mix and left there until 7 days postemergence. Ninety plants of each cultivar were then carefully removed from the pots and dipped for $10 \mathrm{~min}$ in an $8 \times 10^{6} \mathrm{co}-$ 
nidia/ml suspension of $V$. dahliae isolate V18 (race 4A). This isolate is highly aggressive on potato and was isolated from a severely affected potato plant in Wisconsin. Additionally, 10 plants of each cultivar served as controls and were treated similarly to the inoculated plants, except that the roots were dipped in sterile deionized water rather than a suspension of $V$. dahliae conidia. Furthermore, five additional plants of each cultivar were inoculated with $V$. dahliae isolate $\mathrm{V} 18$ and treated similarly, but were raised to maturity, along with noninoculated checks, to confirm expression of typical PED symptoms. Inoculations were performed on 7 March and 3 May for trial 1 and trial 2, respectively.

After dipping in the conidial suspension or water, all plants were transferred to 15 $\mathrm{cm}$ pots filled with the potting mix described above. All 200 plants in each of the two trials were fertilized at the time of transfer to the commercial potting mix using 20-20-20 commercial fertilizer (J. R. Peters, Allentown, PA). Growing conditions were $25 \pm 2^{\circ} \mathrm{C}$ during the day and 18 $\pm 2{ }^{\circ} \mathrm{C}$ at night, with a 16 -h photoperiod and water as needed. Plants were grown in $15-\mathrm{cm}$ plastic pots, which were placed in plastic flats holding 12 plants of one cultivar each. The position of individual flats was arbitrarily assigned in the greenhouse. The two trials were conducted using the same seed and following the same procedures described above.

Sample collection. Fifteen plants of each cultivar were destructively sampled biweekly during the 10 weeks of the trial, starting 2 weeks after inoculation (total 75 inoculated plants). Samples were collected at weeks 2, 4, 6, 8, and 10 after inoculation, thus providing 30 basal stem cuttings (15 per cultivar) every 2 weeks. Samples 5 $\mathrm{cm}$ long were collected from the base of the stem and cut into small pieces $(0.2 \mathrm{~cm})$ divided into two groups. All basal stem cuttings were surface-disinfested in $20 \%$ bleach for $5 \mathrm{~min}$ before processing. One group was squeezed to release $150 \mu$ l of sap, which was used for the CFU quantification assay, by spreading onto petri dishes filled with NPX semi-selective medium. Petri dishes were incubated at room temperature in the dark for 2 weeks. The second group was ground to a powder in liquid nitrogen, using a mortar and pestle, and two 50-mg aliquots were used for DNA extraction.

Apical cuttings from the remaining 15 inoculated plants of each cultivar were taken every 2 weeks starting 2 weeks after inoculation on the same dates the basal stems were sampled. A 1-cm-long portion $(\sim 100 \mathrm{mg})$ of the growing tip of each plant was excised with a sterile blade. The axillary bud just below the apex was then left to grow and the sampling was repeated after 2 weeks. Fifteen apical samples were collected from each cultivar every 2 weeks, and DNA extractions were performed on two aliquots of each apical sample, as described above. Only Q-PCR quantification was performed on apical samples because of insufficient plant material to conduct the plating assay described above. Compared with nonsampled growing plants of the same age, the latter plants were about $20 \%$ smaller and more branched, because of the removal of dominant apical meristem.

CFU quantification. Two 100- $\mu \mathrm{l}$ aliquots of squeezed sap were spread onto one petri plate filled with NPX medium. Colonies were counted using a dissecting microscope after a 2-week incubation in the dark at room temperature.

DNA extraction. All DNA samples were extracted using the FastDNA kit (MP Biomedicals, Irvine, CA) following the manufacturer's recommendations. Subsequently, DNA extracts were subjected to a cleanup using AMPure magnetic beads to eliminate remaining potential PCRinhibitors. Total DNA amounts were estimated by gel electrophoresis (1\% agarose), by comparison to a standardized quantification DNA ladder (High DNA Mass ladder, Invitrogen, Carlsbad, CA). Samples were stored at $4^{\circ} \mathrm{C}$ until used.

Q-PCR amplifications. Q-PCR amplifications were conducted in triplicate, in a Bio-Rad iCycler iQ real-time PCR system using the iQ Supermix SYBR-Green (BioRad) with $\sim 1$ ng of DNA and $200 \mathrm{nM}$ of each primer in $20 \mu \mathrm{l}$ total volume. The following amplification protocol was used: initial denaturation of $3 \mathrm{~min}$ at $95^{\circ} \mathrm{C}$, then 40 cycles of $95^{\circ} \mathrm{C}$ for $10 \mathrm{~s}$ and $63^{\circ} \mathrm{C}$ for 35 s. Melt curve analysis was used to distinguish potential primer dimers and nonspecific amplification products. $V$. dahliae DNA was amplified using the previously described primers VertBt-F (AAC AAC AGT CCG ATG GAT AAT TC) and VertBt-R (GTA CCG GGC TCG AGA TCG) (2). The absence of PCR inhibitors in DNA samples was assessed by spiking each DNA sample with 10 pg of Phytophthora infestans DNA (isolate US-8 940480) and computing the $\Delta \mathrm{Ct}$ value $\left(\Delta \mathrm{Ct}=\mathrm{Ct}_{\text {sample }}-\mathrm{Ct}_{\text {control }}\right)(9)$. In all amplifications, DNA from pure cultures of each $V$. dahliae was used as the positive control and a no-template water sample as the negative control. The infection coefficient (IC), which is the ratio of $\mathrm{Ct}_{\text {host }} / \mathrm{Ct}_{\text {pathogen }}$ (28), was computed for each sample tested. Potato DNA amounts were quantified using previously described primers PotAct-F (TGA ACA CGG AAT TGT CAG CA) and PotAct-R (GGG GTT AAG AGG GGC TTC AG) (3).

Data analysis. The IC and CFU data generated from the quantification of $V$. dahliae in basal samples were analyzed in a two-way ANOVA, using Proc GLM in SAS (SAS Institute, Cary, NC). The impact of the date of sampling (weeks after inoculation), cultivar, and the interaction of these two variables were tested. Least square means separation was performed on the significant cultivar*week interactions. No significant differences were observed between the two trials.

Data from the quantification of $V$. dahliae in apical samples were analyzed in a repeated time measures framework, using Proc Mixed in SAS (17). One of the essential criteria for the ANOVA is the independence of variables, which in this situation was not met, as the same plants were sampled every 2 weeks for 10 weeks. Hence, we opted to use a mixed linear model approach, where sampling dates were random factors and cultivars were fixed factors. Data analysis was performed under three different covariance structure models: compound symmetry, unstructured, and autoregressive 1 . The model that produced the lowest Akaike information criterion (AIC) value is considered the model with the best fit. Least squares mean separations were performed in the model with the best fit. No significant differences were observed between the two trials.

\section{RESULTS}

V. dahliae was detected in stem bases of inoculated plants 2 weeks after inoculation (Fig. 1A), and in the apices after 4 weeks (Fig. 2). Conversely, no evidence of the presence of the fungus was found in noninoculated control plants, throughout the 10 weeks of each experimental trial. As early as 2 weeks after inoculation, cv. Russet Norkotah showed a significantly higher incidence of basal infection with $V$. dahliae (93 and 86.7\% in trials 1 and 2, respectively) compared with cv. Ranger Russet ( 0 and $26.7 \%$ in trials 1 and 2, respectively). Early dying symptoms were not observed on cv. Ranger Russet throughout the study, while symptoms were observed on cv. Russet Norkotah plants as early as week 4. These symptoms included wilting and chlorosis of foliage, in addition to a marked reduction in canopy growth compared with controls. Subsequently, inoculated plants of cv. Russet Norkotah died 2 to 3 weeks earlier than noninoculated controls.

Two weeks after inoculation, the incidence of basal $V$. dahliae infections was significantly higher $(P=0.012)$ in cv. Russet Norkotah compared with cv. Ranger Russet (Fig. 1A). Pathogen incidence in basal samples of cv. Russet Norkotah reached $86.7 \% 2$ weeks after inoculation, and it reached $100 \%$ at 4 weeks after inoculation (Fig. 3). Conversely, incidence was $26.7 \%$ in cv. Ranger Russet at week 2, and gradually increased to above $80 \%$ after week 8 (Fig. 3).

Colonization of basal areas of cvs. Russet Norkotah and Ranger Russet differed significantly over time, with cv. Russet Norkotah exhibiting higher IC and CFU values across all 10 weeks (Fig. 1A). At week 2, the mean IC for cv. Russet Norko- 
tah was 0.58 , while it was 0.17 for $\mathrm{cv}$. Ranger Russet. Values increased consistently and reached 0.99 in cv. Russet Norkotah, while they remained lower than 0.60 in cv. Ranger Russet (Fig. 1A). Additionally, both cultivars were significantly different from each other $(P<0.0001)$ across all sampling dates. No significant differences were observed between $\mathrm{CFU}$ and IC values using a Spearman correlation test $(P<0.0001)$. Nevertheless, the variability in CFU counts was higher than IC values (39 compared with $11.1 \%$, respectively) (Fig. 1). For the remainder of the analyses, only Q-PCR results were utilized. Strong correlations $\left(r^{2}>95 \%\right)$ were found between sampling dates and level of colonization of both cultivars. Colonization increased steadily from week 2 through week 10 in cv. Russet Norkotah (Fig. 1A). Colonization in cv. Ranger Russet was not significantly different between weeks 2 and 4, and it increased and reached a plateau in weeks 6,8 , and 10 (Fig. 1A).

IC values were zero at week 2 in apical samples from both cultivars (Fig. 2), even though basal infections had occurred at that point (Fig. 1). Apical samples from cv. Russet Norkotah were colonized by week 4 (mean IC $=0.64$ ), and IC values increased steadily to reach 0.97 by week 10 (Table 1, Fig. 2). In comparison, no $V$. dahliae was detected in apices of $\mathrm{cv}$. Ranger Russet until week 6 (mean IC = 0.66 ), and it fluctuated slightly between weeks 6,8 , and 10 (Table 1, Fig. 2). The compound symmetry covariance evaluation model produced the lowest Akaike's criterion (AIC $=65.5)$ in the repeated measures analyses, hence mean separations using the least square means were performed under this model (Table 1). The interaction of cultivar with sampling date (cultivar*week) had a significant impact on the colonization of the apices $(P<$ 0.0001). Apices from cv. Russet Norkotah exhibited significant amounts of colonization by week 4, while cv. Ranger Russet exhibited similar colonization at week 6 (Fig. 2). Colonization was significantly different between cultivars at all sampling dates, except for week 2, where no pathogen DNA was detected (Fig. 2).

\section{DISCUSSION}

We compared the dynamics of colonization by $V$. dahliae of two potato cultivars with varying responses to potato early dying, in particular Russet Norkotah (highly susceptible) and Ranger Russet (moderately resistant) $(1,20,26)$. In the past, colonization of potato tissues has been quantified using culture media-based tests, relying on the ability of $V$. dahliae propagules to germinate in vitro. We utilized colony counts and a quantitative realtime PCR assay to describe the colonization dynamics and spatial progression of potato cultivars.
V. dahliae was capable of infecting and colonizing both cultivars. Russet Norkotah plants consistently exhibited higher colonization rates of both basal and apical regions compared with cv. Ranger Russet (Figs. 1 and 2). PED is particularly severe on cv. Russet Norkotah, leading to a premature senescence of potato vines, as well
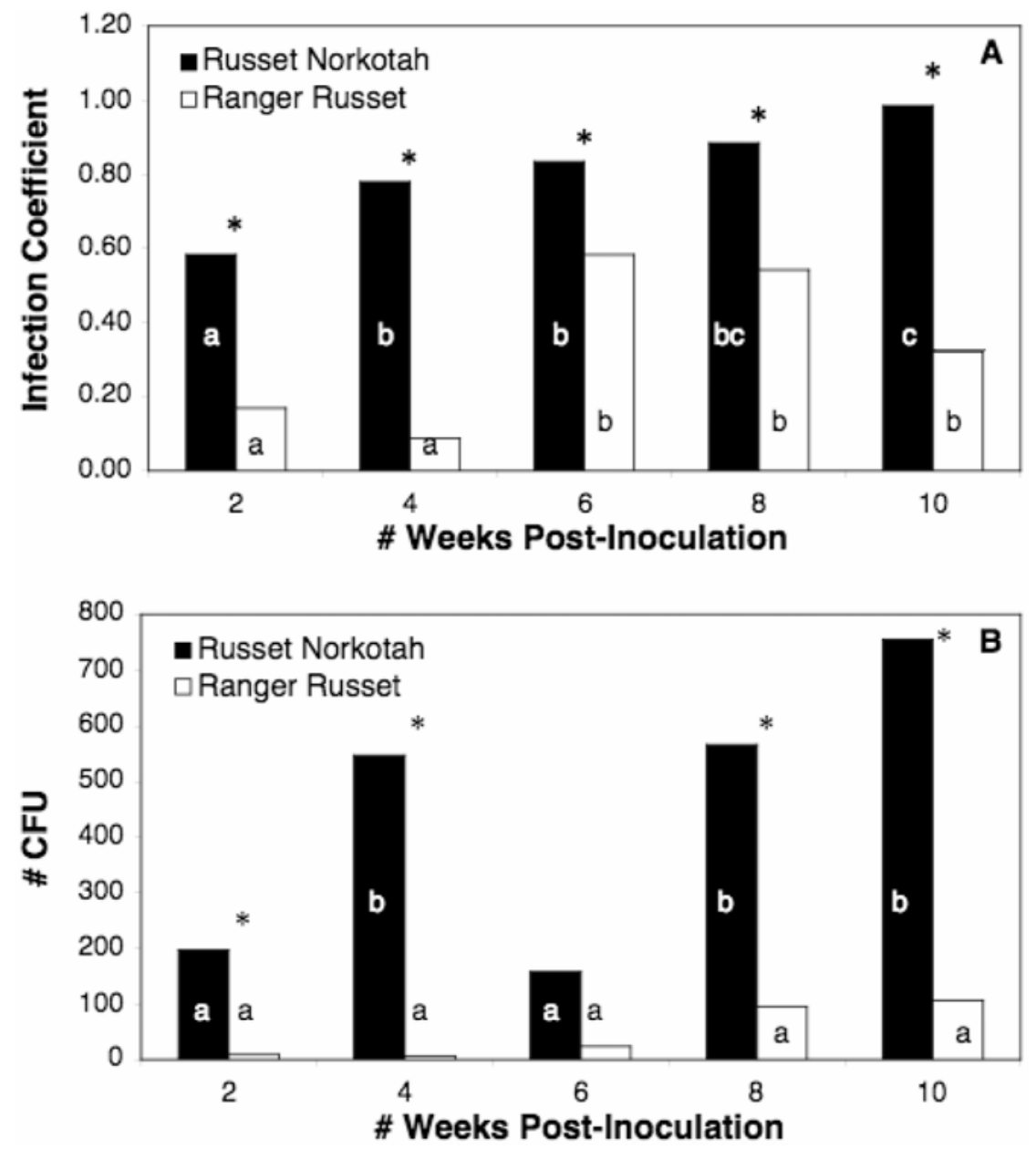

Fig. 1. Colonization by Verticillium dahliae of stem bases in cvs. Ranger Russet (moderately resistant) and Russet Norkotah (highly susceptible) over 10 weeks as measured with a quantitative real-time polymerase chain reaction (Q-PCR) using infection coefficients (A) and NPX plating using CFU (B). Sampling dates within each cultivar sharing the same letter are not significantly different from each other using Fisher's protected LSD test. Asterisks indicate that the two cultivars are statistically significantly different at the sampling date using Fisher's protected LSD test $(\alpha=0.05)$.

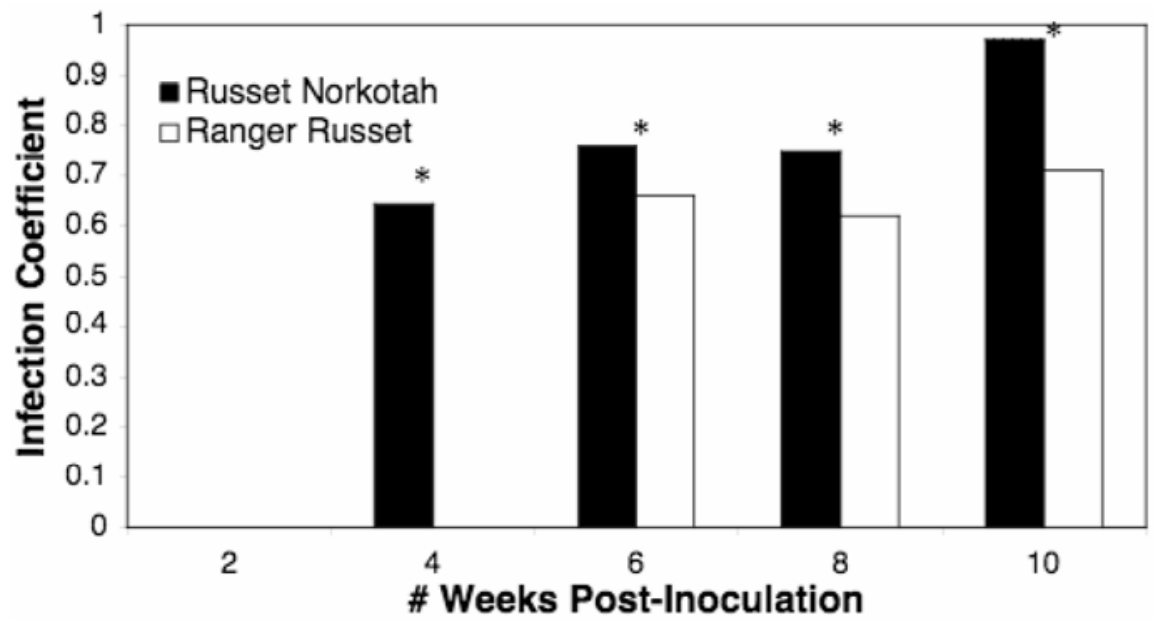

Fig. 2. Colonization by Verticillium dahliae of apices in cvs. Ranger Russet and Russet Norkotah over 10 weeks as measured with a quantitative real-time polymerase chain reaction (Q-PCR) using infection coefficients. Sampling dates with an asterisk are statistically significantly different using a least square means separation test $(\alpha=0.05)$. 
as a significant reduction in yield and number of U.S. No. 1 tubers $(10,26)$. In comparison, cv. Ranger Russet is described as moderately resistant to the disease (26) with adequate tolerance to moisture stress and limited pathogen colonization (1). We found that $V$. dahliae colonized the basal areas of both cultivars as early as 2 weeks after inoculation. But, while cv. Russet Norkotah reached a mean IC close to 0.60 , the mean IC for $\mathrm{cv}$. Ranger Russet was significantly lower at less than 0.20 (Fig. 1A). During the 10 weeks of the study, IC values in cv. Russet Norkotah increased to reach almost 1.0, where the DNA amount of the pathogen and host were even. Conversely, IC values for cv. Ranger Russet increased after week 4 , but reached a plateau between weeks 6 and 10, with the highest mean IC reaching 0.60 (Fig. 1A). At every sampling date, mean IC values were significantly different from each other. Such a finding is in agreement with the described response of each cultivar to potato early dying. Additionally, we found high positive correlations between the date of sampling and IC values, with coefficient correlations in excess of $95 \%$. Davis et al. (7) found a comparable correlation $\left(R^{2}=87.6 \%\right)$ between CFU counts and the date of sampling in naturally infested plots, starting 2 months after planting. In this study, we sampled inoculated plants starting 25 days after planting (2 weeks after inoculation), before any disease symptoms are normally apparent.

$V$. dahliae was detected in the apical portions of cv. Russet Norkotah at week 4, where the mean IC value exceeded 0.60, while the fungus was detected in cv. Ranger Russet at week 6 and the mean IC value exceeded 0.60 (Fig. 2). The colonization of apices increased with time in cv. Russet Norkotah where the mean IC value reached 0.97. Significant differences were observed among most sampling dates in cv. Russet Norkotah, with the exception of weeks 6 and 8, after which IC values increased significantly again (Table 1 ). In cv. Ranger Russet, colonization ceased to increase significantly after the detection of the pathogen in week 6 (Table 1 and Fig. $2)$. The significant decrease in IC values between weeks 6 and 8 in cv. Ranger Russet $(P=0.0108)$ may be attributed to a lag phase in the growth of the inoculum in newly colonized tissues, rather than a decrease in general colonization of the plant. This seems to be substantiated by the lack of significant differences between weeks 6 and 10 (Table 1). The two cultivars were significantly different from each other at all sampling dates except week 2, where no $V$. dahliae DNA was detected. In a previous study (2), we detected $148 \mathrm{fg}$ of $V$. dahliae DNA, which is the equivalent of five nuclei. Such a level of sensitivity indicates an absence of the pathogen in the apical tissues sampled in week 2 in both cultivars and week 4 in cv. Ranger Russet, rather than a failure to detect the pathogen.

A previous study (8) showed that no less than $525 \mathrm{CFU} / \mathrm{g}$ of tissue were needed for a significant correlation with disease incidence. In this study, we found that IC values around 0.80 in the basal samples, and above 0.60 in apical samples, were correlated with symptom appearance in $\mathrm{cv}$. Russet Norkotah. Meanwhile, in cv. Ranger Russet, IC values never exceeded 0.60 in basal samples, and 0.70 in apical samples, and no symptoms of early dying

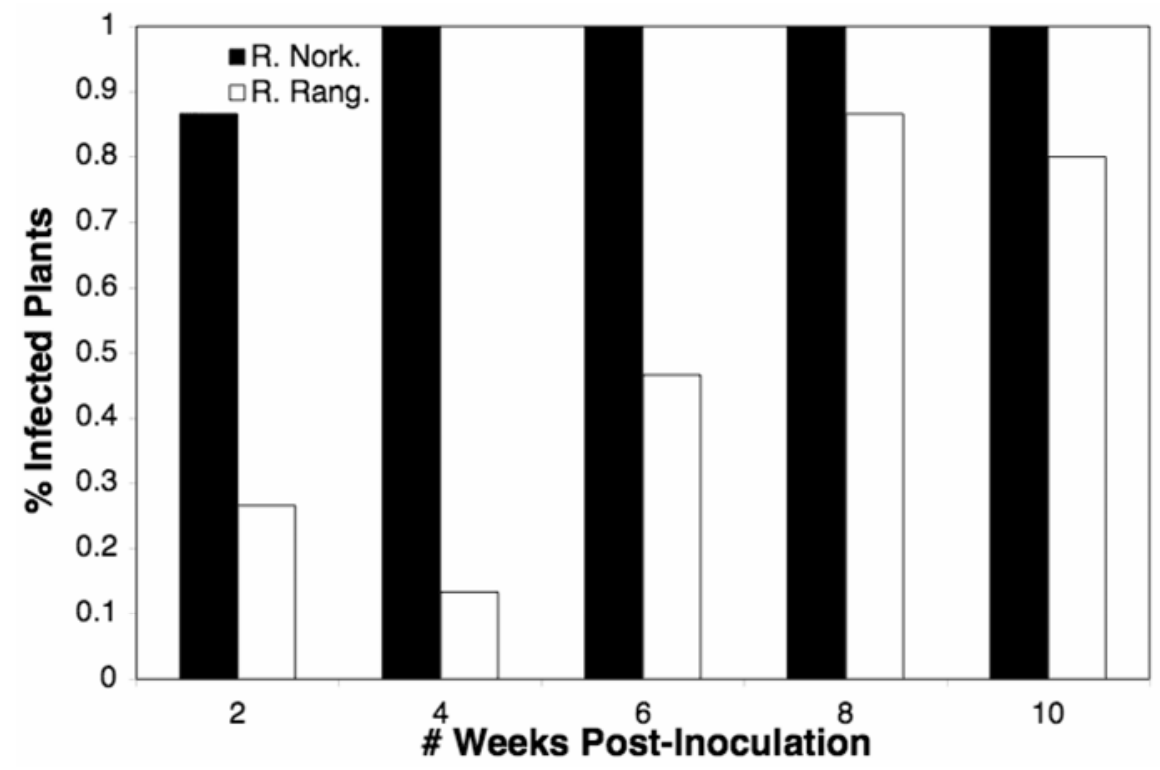

Fig. 3. Comparison of the fortnightly infection incidence over 10 weeks after inoculation by Verticillium dahliae, between the moderately resistant cv. Ranger Russet (white bars) and the highly susceptible cv. Russet Norkotah (black bars), based on the detection of $V$. dahliae DNA in basal or apical plant tissues using quantitative real-time polymerase chain reaction (Q-PCR). Plantlets of both cultivars were inoculated by root immersion in an $8 \times 10^{6}$ conidia/ml suspension on the same date in the greenhouse. could be observed. Additionally, mean $\mathrm{CFU}$ counts never exceeded $100 \mathrm{CFU} / \mathrm{g}$ in basal samples of cv. Ranger Russet, while they were more than $700 \mathrm{CFU} / \mathrm{g}$ in $\mathrm{cv}$. Russet Norkotah (Fig. 1B).

Over the five sampling dates, we found a $97 \%$ correlation between basal and apical IC values in cv. Russet Norkotah, and $85 \%$ in cv. Ranger Russet. These high correlations indicate that the colonization of plant tissues in cv. Ranger Russet goes largely unhampered, while symptom expression does not occur as readily. Nevertheless, the mechanisms leading to the differential expression of early dying symptoms remain unclear, but are perhaps associated with the speed of colonization of apical tissues after the basal areas, and the level of colonization attained. Moreover, $V$. dahliae incidence in cv. Russet Norkotah was higher than $85 \%$ at week 2, compared with $25 \%$ in cv. Ranger Russet (Fig. 3). Incidence reached $100 \%$ in cv. Russet Norkotah by week 4 , while it only reached $85 \%$ by week 10 in cv. Ranger Russet. Hence, it is probable that cv. Russet Norkotah is more susceptible than cv. Ranger Russet because of how fast $\mathrm{V}$. dahliae colonizes and establishes a higher population density in potato plants. From the data presented here, we suggest that breeders examine plant material 6 weeks after inoculation with $V$. dahliae conidia (67 days after planting), when the apices become colonized.

In this study, we investigated, using realtime quantitative PCR, the colonization

Table 1. Least square mean comparisons of infection coefficient (IC) values from apical samples between the five sampling dates for each cultivar

\begin{tabular}{llc}
\hline $\begin{array}{c}\text { Cultivar } \\
\text { Weeks compared }\end{array}$ & Estimate ${ }^{\mathrm{a}}$ & $\begin{array}{c}\text { Standard } \\
\text { error }\end{array}$ \\
\hline Russet Norkotah & & \\
2 and 4 & $-0.30 * *$ & 0.106 \\
2 and 6 & $-0.76 * * *$ & 0.106 \\
2 and 8 & $-0.64 * * *$ & 0.112 \\
2 and 10 & $-0.33 * *$ & 0.103 \\
4 and 6 & $-0.46 * * *$ & 0.106 \\
4 and 8 & $-0.34 * *$ & 0.112 \\
4 and 10 & $-0.33 * * *$ & 0.080 \\
6 and 8 & 0.12 & 0.112 \\
6 and 10 & $0.43 * * *$ & 0.103 \\
8 and 10 & $0.32 * *$ & 0.108 \\
Ranger Russet & & \\
2 and 4 & 0.00 & 0.106 \\
2 and 6 & $-0.40 * *$ & 0.106 \\
2 and 8 & $-0.61 * * *$ & 0.175 \\
2 and 10 & $-0.57 * * *$ & 0.103 \\
4 and 6 & $-0.40 * * *$ & 0.106 \\
4 and 8 & $-0.61 * * *$ & 0.175 \\
4 and 10 & $-0.57 * * *$ & 0.103 \\
6 and 8 & $0.28 *$ & 0.106 \\
6 and 10 & -0.17 & 0.103 \\
8 and 10 & $-0.45 * * *$ & 0.103 \\
\hline
\end{tabular}

a Negative values indicate an increase in mean IC values, and positive values indicate a decrease in mean IC values (* significant at $\alpha=$ $0.05, * *$ significant at $\alpha=0.01$, *** significant at $\alpha=0.001$ ). 
and spatial progression by $V$. dahliae of two potato cultivars with varying responses to PED. The pathogen was detected in basal samples of cvs. Russet Norkotah (highly susceptible) and Ranger Russet (moderately resistant) 2 weeks after inoculation of plantlets in the greenhouse with conidia of $V$. dahliae isolate V18. Four weeks after inoculation, $V$. dahliae was detected in apical samples of cv. Russet Norkotah, while it was not detected in cv. Ranger Russet until week 6. Colonization of cv. Russet Norkotah consistently increased in both apical and basal samples during the 10 weeks of the experiment, while it reached a plateau after week 6 in cv. Ranger Russet. This study is an attempt at understanding the population dynamics of $V$. dahliae in potato cultivars to explain the process of resistance to PED. This research provides breeders with much needed clarifications regarding the nature of resistance to $V$. dahliae in cvs. Ranger Russet and Russet Norkotah and describes a new time-saving approach to utilize in the selection of breeding lines with enhanced resistance to PED.

\section{ACKNOWLEDGMENTS}

We thank D. A. Johnson and D. A. Halterman for their review of the manuscript and suggestions to improve it. This research was funded by grants from the USDA-CSREES-Potato Research Program and the Wisconsin Potato and Vegetable Growers Association to Z.K.A. and W.R.S. The authors also thank the senior editor and anonymous reviewers of Plant Disease for helping improve this manuscript.

\section{LITERATURE CITED}

1. Arbogast, M., Powelson, M. L., Cappaert, M. R., and Watrud, L. S. 1999. Response of six potato cultivars to amount of applied water and Verticillium dahliae. Phytopathology 89:782788.

2. Atallah, Z. K., Bae, J., Jansky, S. H., Rouse, D. I., and Stevenson, W. R. 2007. Multiplex realtime quantitative PCR to detect and quantify Verticillium dahliae colonization in potato lines that differ in response to Verticillium wilt. Phytopathology 97:865-872.

3. Atallah, Z. K., and Stevenson, W. R. 2006. A methodology to detect and quantify five pathogens causing potato tuber decay using real-time quantitative PCR. Phytopathology 96:1037-1045.
4. Bowden, R. L., and Rouse, D. I. 1991. Effects of Verticillium dahliae on gas exchange of potato. Phytopathology 81:293-301.

5. Bowden, R. L., Rouse, D. I., and Sharkey, T. D. 1990. Mechanism of photosynthesis decrease by Verticillium dahliae in potato. Plant Physiol. 94:1048-1055.

6. Butterfield, E. J., and Devay, J. E. 1977. Reassessment of soil assays for Verticillium dahliae. Phytopathology 67:1073-1078.

7. Davis, J. R., Pavek, J. J., and Corsini, D. L. 1983. A sensitive method for quantifying Verticillium dahliae colonization in plant tissue and evaluating resistance among potato genotypes. Phytopathology 73:1009-1014.

8. Davis, J. R., Pavek, J. J., Corsini, D. L., Sorensen, L. H., Schneider, A. T., Everson, D. O., Westermann, D. T., and Huisman, O. C. 1994. Influence of continuous cropping of several potato clones on the epidemiology of Verticillium wilt of potato. Phytopathology 84:207-214

9. Gao, X., Jackson, T. A., Lambert, K. N., Li, S., Hartman, G. L., and Niblack, T. L. 2004. Detection and quantification of Fusarium solani f. sp. glycines in soybean roots with real-time quantitative polymerase chain reaction. Plant Dis. 88:1372-1380.

10. Hoyos, G. P., Zambino, P. J., and Anderson, N. A. 1991. An assay to quantify vascular colonization of potato by Verticillium dahliae. Am. Potato J. 68:727-742.

11. Jansky, S. H., and Rouse, D. I. 2000. Identification of potato interspecific hybrids resistant to Verticillium wilt and determination of criteria for resistance assessment. Potato Res. 43:239-251.

12. Jansky, S. H., and Rouse, D. I. 2003. Multiple disease resistance in interspecific hybrids of potato. Plant Dis. 87:266-272.

13. Jansky, S., Rouse, D. I., and Kauth, P. J. 2004. Inheritance of resistance to Verticillium dahliae in diploid interspecific potato hybrids. Plant Dis. 88:1075-1078.

14. Jeger, M. J., Hide, G. A., VandenBoogert, P., Termorshuizen, A. J., and VanBaarlen, P. 1996. Pathology and control of soil-borne fungal pathogens of potato. Potato Res. 39:437-469.

15. Johansen, R. H., Farnsworth, B., Nelson, D. C., Secor, G. A., Gudmestad, N., and Orr, P. H. 1988. Russet Norkotah - a new russet-skinned potato cultivar with wide adaptation. Am. Potato J. 65:597-604.

16. Kimpinski, J., Platt, H. W., Perley, S., and Walsh, J. R. 1998. Pratylenchus spp. and Verticillium spp. in New Brunswick potato fields. Am. J. Potato Res. 75:87-91.

17. Littell, R. C., Henry, P. R., and Ammerman, C. B. 1998. Statistical analysis of repeated measures data using SAS procedures. J. Animal Sci. 76:1216-1231.

18. Lynch, D. R., Chen, Q., Kawchuk, L. M., and
Driedger, D. 2004. Verticillium wilt resistan germplasm-release of clone LRC18-21 and derivatives. Am. J. Potato Res. 81:295-297.

19. Mahuku, G. S., Platt, H. W., and Maxwell, P. 1999. Comparison of polymerase chain reaction based methods with plating on media to detect and identify Verticillium wilt pathogen of potato. Can. J. Plant Pathol. 21:125-131.

20. Mohan, S. K., Davis, J. R., Corsini, D. L., Sorensen, L. H., and Pavek, J. J. 1990. Reaction of potato clones and accessions of Solanum spp to Verticillium dahliae Kleb and its toxin. Potato Res. 33:449-458.

21. Mpofu, S. I., and Hall, R. 2003. Accuracy and precision of population estimates of Verticillium dahliae on growth media in quantitative soil assays. Can. J. Bot.-Rev. Can. Bot. 81:294-306.

22. Nicot, P. C., and Rouse, D. I. 1987. Relationship between soil inoculum density of Verticillium dahliae and systemic colonization of potato stems in commercial fields over time. Phytopathology 77:1346-1355.

23. Pavek, J. J., Corsini, D. L., Love, S. L., Hane, D. C., Holm, D. G., Iritani, W. M., James, S R., Martin, M. W., Mosley, A. R., Ojala, J. C., Stanger, C. E., and Thornton, R. E. 1992. Ranger Russet - a long russet potato variety for processing and fresh market with improved quality, disease resistance, and yield. Am. Potato J. 69:483-488.

24. Powelson, M. L., and Rowe, R. C. 1993. Biology and Management of Early Dying of Potatoes. Annu. Rev. Phytopathol. 31:111-126.

25. Rowe, R. C., Davis, J. R., Powelson, M. L., and Rouse, D. I. 1987. Potato early dying: Causal agents and management strategies. Plant Dis. 71:482-489.

26. Rowe, R. C., and Powelson, M. L. 2002. Potato early dying: Management challenges in a changing production environment. Plant Dis. 86:1184-1193.

27. Termorshuizen, A. J., Davis, J. R., Gort, G. Harris, D. C., Huisman, O. C., Lazarovits, G. Locke, T., Vara, J. M. M., Mol, L., Paplomatas, E. J., Platt, H. W., Powelson, M., Rouse, D. I., Rowe, R. C., and Tsror, L. 1998. Interlaboratory comparison of methods to quantify microsclerotia of Verticillium dahliae in soil. Appl. Environ. Microbiol. 64:3846-3853.

28. Valsesia, G., Gobbin, D., Patocchi, A., Vecchione, A., Pertot, I., and Gessler, C. 2005. Development of a high-throughput method for quantification of Plasmopara viticola DNA in grapevine leaves by means of quantitative realtime polymerase chain reaction. Phytopathology 95:672-678.

29. Wheeler, T. A., Rowe, R. C., Riedel, R. M. and Madden, L. V. 1994. Influence of cultivar resistance to Verticillium spp. on potato early dying. Am. Potato J. 71:39-57. 